Journal of Social Sciences 5 (4): 416-422, 2009

ISSN 1549-3652

(C) 2009 Science Publications

\title{
The Effect of Exhausting Exercise Induced Muscular Fatigue On Functional Stability
}

\author{
${ }^{1}$ Khodadad Letafatkar, ${ }^{2}$ Mohammad Hossein Alizadeh and ${ }^{3}$ Mohammad Reza Kordi \\ ${ }^{1}$ Faculty of Physical Education and Sport Science, \\ ${ }^{2}$ Department of Sport Injuries, Faculty of Physical Education and Sport Science, \\ ${ }^{3}$ Department of Exercise Physiology, Physical Education and Sport Science, \\ Tehran University, Iran
}

\begin{abstract}
Problem statement: To quantify the effect of exhausting exercise induced muscular fatigue on functional stability. Approach: About 30 male professional athletes with mean age $23 \pm 2.1$ years old, mean weight $82 \pm 1.3 \mathrm{~kg}$ and mean height $181 \pm 9.7 \mathrm{~cm}$ were selected for participation in this study. In this study Unstable Biodex platform (level 3) used for stability evaluation. Balance performance was quantified as a stability index and the mean deflection of the platform. Running Based Anaerobic Test (RAST test) used for fatigue execution. Results: Results showed no significant ( $>0.05)$ main effect for exercise duration in the stability index, suggesting that balance performance was maintained throughout the competition. Hence there wasn't a significant relationship between lactate acid accumulation and variations of stability indices $(r=-0 / 661, p<0.05)$. Conclusion: Therefore, the improvement of proprioception in the ankle and knee joint is recommended as a prevention role to the injuries in the abovementioned joints.
\end{abstract}

Key words: Balance, fatigue, stability

\section{INTRODUCTION}

Fatigue is a complex phenomenon that can be described as a time-dependent exercise-induced reduction in the maximal force generating capacity of a muscle $^{[1]}$. Alteration in performance tends to vary across sports that are influenced more or less by factors like decreased muscular power and endurance, decreased motor skill performance and mental lapses. It is well known that impairment of performance resulting from neuromuscular fatigue differs according to the type of contraction, the muscular group tested and the exercise duration/intensity ${ }^{[2]}$. Therefore, the muscular fatigue can be the result of a failure in any process involved in the muscular contraction ${ }^{[3]}$. Historically, the potential factors involved in the fatigue development are divided in two categories: the central factors that should provoke the fatigue by a disorder in the neuromuscular transmission between the CNS and the muscular membrane and peripheral factors that would cause an alteration inside the muscle ${ }^{[4-6]}$. Another characteristic of the fatigue is the fact that it depends on the task, that is, its causes vary in a very wide way and it behaves according to the way it is induced ${ }^{[3,7]}$. The muscular fatigue is considered as a predisposing factor to the appearance of injuries ${ }^{[8,9]}$, such as the ankle sprain. Lactic acid accumulation is one of the cases that can be interfering in fatigue protocol and subsequently decreasing the functional stability. Surenkok et al. ${ }^{[1]}$ showed that there was no correlation between lactic acid accumulation and stability changes. It can be concluded that knee muscle fatigue has an adverse effect on balance; however, this impairment was not correlated with the degree of lactic acid accumulation $^{[11]}$. Several authors have been studying the effects of the muscular fatigue on the neuromuscular control ${ }^{[8,14-16]}$, which is related to the proprioceptive afferents that are taken by the peripheral receptors to the upper centers and to the efferent (motor) responses generated with the purpose to keep the dynamic muscular stability ${ }^{[17]}$. Studies have shown that the muscular fatigue causes an adverse change in the proprioception $^{[15,18]}$ a sensorial modality comprising the sensations of the joint movement and positioning ${ }^{[12,38]}$, as well as the postural control ${ }^{[8,14,16,19]}$. Stability is defined as the ability to maintain intervertebral and global torso equilibrium despite the presence of small mechanical disturbances and/or small neuromuscular control errors. Stability of the spine may be impaired by fatigue of the paraspinal muscles ${ }^{[20]}$. Aetiological risk factors

Corresponding Author: Khodadad Letafatkar, Faculty of Physical Education and Sport Sciences, University of Tehran, Tehran, Iran 
associated with joint sprain injury incidence and severity $^{[21]}$ include impaired proprioceptive capacity ${ }^{[22,37]}$ and postural stability ${ }^{[23,39]}$. Rozzi et al. ${ }^{[24]}$ suggested that in the fatigued state the joint may fail to produce the appropriate muscular responses which have a protective function in maintaining joint stability.

The aim of the present study was to examine the influence the effect of exhausting exercise induced muscular fatigue on functional stability.

\section{MATERIALS AND METHODS}

Participants: This study included 30 apparently healthy young male adults (handball and basketball professional players), with mean age $23 \pm 2.1$ years old, mean weight $82 \pm 1.3 \mathrm{~kg}$ and mean height $181 \pm 9.7 \mathrm{~cm}$ matched evenly for age. The protocol used in this study was reviewed and approved by Tehran University's Institutional Review Board prior to participant recruitment and all participants provided written informed consent prior to beginning the study. As assessed by a medical history questionnaire, all subjects were male and free of vestibular disorders and none had suffered a mild head injury or lower extremity injury in the preceding 6 months. Firstly Subjects were tested between 8:00 and 10:00 $\mathrm{h}$, according to the regular training. Participants attended having performed no vigorous exercise in the $24 \mathrm{~h}$ prior to testing and with diet standardized for $48 \mathrm{~h}$ proceeding in each test. Players were required to consume $500 \mathrm{ml}$ of water $2 \mathrm{~h}$ prior to testing to ensure dehydration. Thereafter the subjects consumed no fluid so as to control for the possible influence of hydration status on performance $^{[52]}$. Subjects participated in the RAST test after having a standard breakfast and after having their blood lactate levels measured via the un-preferred hand mid-fingertip blood samples by a certificated lactometer. All subjects completed familiarization trials of the balance task in the rested state on a minimum of previous laboratory visits. The stabilometer trial comprised a (30's eyes closed and 30's eyes opened respectively) double-legged balance task, where the subjects was instructed to keep the dynamic and unstable platform level to the best of their ability. During the balance task each player was instructed to look directly ahead, with slight flexion at the knee, equivalent to the stork stance. No visual feedback was provided to the subject with regard to performance. After measuring the blood lactate the Subjects participated in the RAST test. Before and immediately after the fatigue protocol (RAST test), blood lactic acid levels measured via lactometer (production of Sense Lab Company) then double-leg balance on biodex system measured after the RAST test. Indices of balance were quantified as an overall measure and also in both the anterior-posterior and medio-lateral planes. The same directional indices were quantified for the mean deflection over the duration of the balance task.

\section{Instruments:}

Dynamic postural stability: Participants stood barefoot on a Biodex system (level 3). The Biodex system measured any postural sway resulting from the focal movement by recording the displacement and velocity of the COP. To assess balance and neuromuscular control, this study used a commercially available balance device, the BSS (Biodex Medical Systems, Shirley, NY, USA), which consists of a movable balance platform that provides up to $20^{\circ}$ of surface tilt in a $360^{\circ}$ range of motion. The platform is interfaced with computer software (Biodex, Version 3.1, Biodex Medical Systems) that enables the device to serve as an objective assessment of balance. The measure of postural stability includes the Overall (OA), the Anterior/Posterior (AP) and the Medial/Lateral (ML) stability scores. A high score in the OA index indicates poor balance. The OA stability score is believed to be the best indicator of the overall ability of the subjects to balance the platform ${ }^{[54]}$. We assessed bilateral stance at level 3 (more unstable) with the BSS over a period of (30's eyes closed and 30's eyes opened respectively). Following the recommendations of the previous studies ${ }^{[40,54]}$, level 3 is used as the unstable levels. Subjects were asked to step on the platform of the BSS and assume a comfortable position while maintaining slight flexion in the knees $\left(15^{\circ}\right)$, to look straight ahead and to place arms across the chest. Foot position coordinates were constant throughout the test session. Subjects and controls were trained $1 \mathrm{~min}$ for adaptation to the machine and then 1 min double-leg balance test evaluations were performed. A mean score was calculated from the one test evaluations.

\section{Fatiguing task:}

RAST test: in this study the RAST test used for fatigue compelling in subjects. This test was chosen primarily due to its validity and reliability in relation to the Wingate test and also, because this test requires minimal equipment and training of the assessors and because of its specialty for field based anaerobic activities that are of a repetitive nature. It is easy to perform, provides scores that are easily reproduced and can be used to successfully estimate anaerobic capacity $^{[49]}$. In order to carry out a correct and precise testing process, the subjects stood $70 \mathrm{~cm}$ in the back of 
the starting line (on each side) and the apparatus would let the timer start after the subject passed in front of the first photocell. It was also decided that if in any of the cases the subject's best record was achieved after the second repetition, the test process should be finished and the subject was allowed to have another opportunity to participate in the test. To avoid these unwanted cases, subjects were asked to do each repetition at maximum power and avoid dividing energy between the six repetitions. Also in order to increase the subjects' motivation, the record of each repetition was announced loudly and there were special rewards for three individuals who could achieve the best record, in addition to the payment in consideration of all the participants ${ }^{[49]}$.

Blood samples were collected from unpreferred hand mid-fingertips two times (1) immediately prior to the RAST test (pre-lac), (2) 5 min after the RAST test $(5 \mathrm{lac})$ ) for the purpose of estimating blood lactate using a lactate analyzer (Analox P-LM55, UK) found in an Analox lactate kit supplied by Analox (UK). It should be noted that, the analyzer had been calibrated with known lactate standards (5.0 and $15.0 \mathrm{mM}$ ). Since environmental conditions can affect blood lactate levels and performance ${ }^{[55]}$, air temperature and relative humidity values for the track were recorded $(21.7 \pm 5.9$ and $48.4 \pm 11.9 \%$ ) using an Arco device (Model TC14P; Germany).

Procedure: One-week before the data collection took place; the participants were instructed on how to perform the RAST test and underwent a familiarization session (after performing $400 \mathrm{~m}$ running test). In addition, they were asked to wear comfortable, loosefitting clothing and get an adequate amount of sleep (6$8 \mathrm{~h}$ ) the night before the test ${ }^{[45}$. The clothing, shoes, as well as all equipment used, were consistent for each subject and were recorded to establish controlled experimental conditions.

Experimental protocol: The subjects arrived at the stadium at 8:00 a.m. in a fasted state and a standardized breakfast (approximately $380 \mathrm{kcal}$ ) was served and they lactic and balance tests on BIODEX system (level 3) was taken. Since the RAST test is an all-out test, the subjects warmed up for at least $25 \mathrm{~min}$. The warm-up was standardized for all of the participants ${ }^{[46]}$. During the RAST test, the time of each repetition and the rest intervals was measured by an automatic timing photocell system. Before and 5 min after the RAST test, the balance tests were performed on biodex system.

Statistical analysis: The pre and post-test data's were analyzed with a paired t-test and other data's analyzed by sample t-test An alpha level of (0.05) was used in determining statistical significance using the SPSS program for Windows, version 16.0.

\section{RESULTS}

There wasn't significant relationship between lactate acid accumulation and variations of stability indices $(r=-0 / 661, \mathrm{p}<0.05)$.

\section{DISCUSSION}

The purpose of this study was to quantify the effect of exhausting exercise induced muscular fatigue on functional stability. One of the factors evaluated in this study was the (overall, ant-post and med-lat) stability index throughout fatigue training that results revealed that there aren't any significant main effects for time, such that double legged balance performance was maintained throughout the trial (Table 1).

The results of this study showed that there weren't significant differences in overall, ant- post and med- lat stability index $(\mathrm{p}=0.195, \mathrm{p}=0.087$ and $\mathrm{p}=0.130$ respectively), platform ant- post and med- lat deflection ( $\mathrm{p}=0.091, \mathrm{p}=0.152$ respectively). Thus, According to These results, we can conclude that muscular fatigue probably can't affect functional stability in professional athletes.

Few studies have directly measured the effects of sport induced specific fatigue on functional stability. Greig et al. ${ }^{[48]}$ indicated that sport specific fatigue couldn't affect stability index by times ${ }^{[36]}$. Schieppati ${ }^{[43]}$ and Torbjorn ${ }^{[10]}$ completed a study in fatigue mechanisms; they found that fatigue can alter the stability indices. APA mechanism can compensate the balance decreases. In other words, early APA onset may reflect an attempt by the CNS to compensate for a reduction in the force producing capability of a fatigued muscle by producing a muscular impulse under a fatigued state that is similar to that required in a nonfatigued state. This impulse would enable postural control to be maintained in the presence of fatigue ${ }^{[44]}$. According to this study results there wasn't significant relationship between lactate acid accumulation and variations of stability indices. We concluded that lactate acid accumulation couldn't affect stability index.

\begin{tabular}{|c|c|c|c|}
\hline \multirow[b]{2}{*}{ Variables } & \multicolumn{3}{|l|}{ Statistics } \\
\hline & Mean differences & $\mathrm{t}$ & sig \\
\hline Overall stability index & -0.258 & -1.184 & 0.195 \\
\hline Ant-post stability index & -0.241 & -2.117 & 0.087 \\
\hline Med-lat stability index & -0.268 & -1.112 & 0.130 \\
\hline Lactate (after $5 \mathrm{~min}$ ) & 0.272 & 3.111 & $0.028 *$ \\
\hline Ant-post platform deflection & -0.313 & -1.098 & 0.091 \\
\hline Med-lat platform deflection & -0.273 & -2.231 & 0.152 \\
\hline
\end{tabular}




\section{J. Social Sci., 5 (4): 416-422, 2009}

One reason for this result is that the professional handball and basketball player often training in the lactate threshold (based on energy systems), that this case can improved they body resistance for lactate acid accumulation and therefore the lactate acid accumulation couldn't affect stability index. Also Surenkok et al. ${ }^{[11]}$ showed that there was no correlation between lactic acid accumulation and stability changes. It can be concluded that knee muscle fatigue has an adverse effect on balance; however, this impairment was not correlated with the degree of lactic acid accumulation $^{[11]}$. The effectiveness of sports injury prevention programs has been well documented both in epidemiological studies that showed a decrease of athletic injuries ${ }^{[40,41]}$. In the context of the multi-sensory control of balance, when the availability or the reliability of input from a particular body location decreases, it is conceivable the central nervous system to increase the weighting of input from other locations that provide reliable information for maintaining stable posture $^{[42]}$. The Biodex stabilometer task provides a measure of deviation of a surface which pivots about a central axis, such that it is free to move simultaneously in the anterioposterior and medio-lateral plane. The deviation of the platform is directly related to the stability of the player, such that greater body movement creates greater deviation in the platform and subsequently a high stability index. With no significant main effect for exercise duration it might be interpreted that the intermittent competition had no effect on single legged balance performance. Consideration of the directional stability indices reveals that stability was greater in the medio-lateral plane than in the anteriorposterior plane, until the post-exercise measure. This might reflect the anatomical configuration of boney and soft tissue structures ${ }^{[26]}$. Instability in the medio-lateral plane is likely to pose a greater risk for joint injury and the finding of no fatigue effect with exercise duration suggests that joint stability was not compromised. However, consideration of the mean deflection of the platform over the duration of the task indicates that a change of strategy might have been employed. In each trial the mean deflection was lateral to the centre of the platform, as expected. However, at the end of each half the mean deflection in the anterior-posterior direction was seen to increase in the anterior direction. This toes down rotation of the platform is indicative of greater plantar flexion at the ankle. In a more functional setting plantar-flexion of the ankle reduces the base of support and increases the risk of ankle sprain injury due to the additional rotational and transverse movements allowed towards the more open packed position of the ankle joint ${ }^{[26]}$. The anterior deflection might also be achieved by increased knee or hip flexion to move the centre of mass forward. Injury risk might be increased when placing greater reliance on knee or hip strategies to maintain balance, due to changes in muscular recruitment patterns. This interpretation is supported by the observations of Adlerton et al. ${ }^{[27]}$ who reported a post-fatigue change in postural control strategy, where the habitual strategy changed from ankle to hip following localized muscle fatigue of the calf. The modifications made in the postural control pattern produce compensatory corrections around the joints to maintain functional stability. However, whilst balance performance is maintained, the fatigue-induced alterations in strategy might make the player more susceptible to injury. The alteration in balance strategy during the latter stages of each half suggests that functional joint stability is impaired during the latter stages of each half. This finding supports epidemiological observations of temporal patterns in ankle sprain incidence during soccer match-play ${ }^{[28]}$. The nature of the change in balance strategy, i.e., an inverted ankle that becomes increasingly plantar-flexed during the latter stages of match-play, is also correlated to injury epidemiology. Lateral ankle sprain is a common injury, accounting for $25-50 \%$ of all injuries in sports $^{[29]}$ and $17 \%$ of soccer injuries ${ }^{[30]}$. Woods et al. ${ }^{[28]}$ reported that $77 \%$ of all ankle ligament sprain injuries were localized to the lateral complex. An inversion force applied to the foot with the ankle in plantarflexion was described as a common mechanism of injury to the lateral ligament. This scenario is analogous to cutting maneuvers and multi-directional jumping inherent in handball activities. It is not possible to conclusively state the mechanism driving the change in balance strategy during the latter stages of each half. Fatigued muscles have been shown to exhibit extended latency in firing ${ }^{[28]}$, electromechanical delay ${ }^{[32,33]}$ and slower muscle reaction time ${ }^{[34]}$. The subsequent impairment of the dynamic stabilizing function of muscles is a primary factor in the non-contact etiology of joint ligamentous injury ${ }^{[35]}$.

Further support for the proposition that lactic acid accumulation is advantageous during muscle activity is provided by the properties of the two major Monocarboxylate Transporters (MCTs), which play a major role in the regulation of intracellular $\mathrm{pH}$ and lactate concentration during intense muscle activity. The MCT4 isoform that is predominantly and abundantly expressed in fasttwitch glycolytic fibers, the major producer of lactic acid, has a relatively high dissociation constant (low affinity) $\mathrm{Km}$ of 20-35 mM, whereas the MCT1 isoform, which is predominantly expressed in slow-twitch oxidative fibers, has a $\mathrm{Km}$ of 
3-5 $\mathrm{mM}^{[51]}$. The high $\mathrm{Km}$ of MCT4 for lactate explains why lactic acid is allowed to accumulate in the fasttwitch glycolytic muscle during exercise, causing acidification of the myoplasm. This must be beneficial for the muscle because otherwise the muscle would have expressed the low Km MCT isoform. The lower $\mathrm{Km}$ value for lactate of MCT1 isoform in the slowtwitch, oxidative muscle fibers provide a higher affinity uptake mechanism for lactate and protons to be used in these fibers as a respiratory fuel. Finally, we note that a rise in blood lactate (the "lactate threshold") can indeed be used as an indicator of exhaustion. However, although lactate may well increase when muscle performance declines, lactate is not the cause of the decline. Lactate rises in the blood when the muscle cells are using ATP faster than they re-synthesize it aerobically in the mitochondria. But it is the other changes occurring in the muscle, not the lactic acid accumulation, which cause the fatigue. Acidity associated with lactic acid accumulation actually helps delay the onset of muscle fatigue that would otherwise ensue from the other effects of vigorous activity ${ }^{[50]}$. The effectiveness of sports injury prevention programs has been well documented both in epidemiological studies that showed a decrease of athletic injuries ${ }^{[4,53]}$. In the context of the multi-sensory control of balance, when the availability or the reliability of input from a particular body location decreases, it is conceivable the central nervous system to increase the weighting of input from other locations that provide reliable information for maintaining stable posture ${ }^{[8]}$.

\section{CONCLUSION}

This leads to the conclusion that, despite muscular fatigue, ankles appear to be more stable after exercise. Does exercise induce an increase in afferent/efferent nerve impulses to and from muscle spindles around the ankle leading to improved joint position sense? It is suggested that handball and basketball players perform proprioception drills both in the rested and exercised state.

\section{ACKNOWLEDGEMENT}

Gratitude is expressed to the subjects that participated in this study as well as to each of the assistants who were instrumental in the collection of the data. This study was funded by a product grant from the University of Tehran (Tehran, Iran). The researchers independently collected, analyzed and interpreted the results and have no financial interests in the results of this study. Also, dissemination of the results in this study does not constitute endorsement by the researchers or their institutional affiliations.

\section{REFERENCES}

1. Gandevia, S.C., 2001. Spinal and supraspinal factors in human muscle fatigue. Physiol. Rev., 81: 1725-1789. PMID: 11581501

2. Millet, G.Y. and R. Lepers, 2004. Alterations of neuromuscular function after prolonged running, cycling and skiing exercises. Sports Med. 34: 105-116. PMID: 14965189

3. Gandevia, S.C., 1998. Neural control in human muscle fatigue: Changes in muscle afferents, motoneurones and motor cortical drive. Acta Physiol. Scand, 162: 275-283. PMID: 9578373

4. Giannesini, B., P.J. Cozzone and D. Bendahan, 2003. Non-invasive investigations of muscular fatigue: Metabolic and electromyographic components. Biochimie, 85: 873-883. PMID: 14652176

5. Kent-Braum, J.A., 1999. Central and peripheral contributions to muscle fatigue in humans during sustained maximal effort. Eur. J. Applied Physiol., 80: 57-63. PMID: 10367724

6. Schillings, M.L., W. Hoefsloot, D.F. Stegeman and M.J. Zwarts, 2003. Relative contributions of central and peripheral factors to fatigue during a maximal sustained effort. Eur. J. Applied Physiol., 90: 562-268. http://cat.inist.fr/?aModele $=$ afficheN\&cpsidt $=15436367$

7. Enoka, R.M., 2000. Bases Neuromecânicas da Cinesiologia. 2nd Edn., São Paulo, Manole, ISBN: 8520407951, pp: 320-344.

8. Gefen, A., M. Megido-Ravid, Y. Itzchak and M. Arcan, 2002. Analysis of muscular fatigue and foot stability during high-heeled gait. Gait Posture, 15: 56-63. DOI: 10.1016/S0966-6362(01)00180-1

9. Gorelick, M., J.M.M. Brown and H. Groeller, 2003. Short-duration fatigue alters neuromuscular coordination of trunk musculature: Implications for injury. Applied Ergon., 34: 317-25.

10. Torbjorn, Ledin, P. A. Fransson and M. Magnusson, 2006. Effects of postural disturbances with fatigued triceps surea muscles or with $20 \%$ additional body weight. Gait Posture, 19: 184-193. DOI: 10.1016/S0966-6362(03)00061-4

11. Surenkok, A., E. Scedil, K. Isler, A. Aytar, Z. Gultekin and M.N. Akman, 2006. Effect of knee muscle fatigue and lactic acid accumulation on balance in healthy subjects. Isokinet. Exerc. Sci., 14: 301-306.

12. Frontera, 2000. Rehabilitation in Sport Injury. Wiley-Blackwell, ISBN: 0632058137, pp: 270-291. 
13. Vachon, A.J., R.D. Bassett and S. Clarke, 1999. Validity of the heart rate deflection point as a predictor of lactate threshold during running. J. Applied Physiol., 87: 452-459. http://jap.physiology.org/cgi/content/full/87/1/452/T2

14. Chabran, E., B. Maton and A. Fourment, 2002. Effects of postural muscle fatigue on the relation between segmental posture and movement. J. Electromyogr. Kinesiol, 12: 67-79.

15. Lee, H.M., J.J. Liau, C.K. Cheng, C.M. Tan and J.T. Shih, 2003. Evaluation of shoulder proprioception following muscle fatigue. Clin. Biomech (Bristol, Avon), 18: 843-847. http://www.ncbi.nlm.nih.gov/pubmed/14527811

16. Yaggie, J.A. and S.J. McGregor, 2002. Effects of isokinetic ankle fatigue on the maintenance of balance and postural limits. Arch. Phys. Med. Rehabil., 83: 224-228. http://www.ncbi.nlm.nih.gov/pubmed/11833026?d opt=Abstract

17. Lephart, S.M. and F.H. Fu, 2000. Proprioception and neuromuscular control in joint stability. 1st Edn., Human Kinetics, ISBN: 10: 0880118644, pp: 439.

18. Miura, K., Y. Ishibashi, E. Tsuda, Y. Okamura, H. Otsuka and S. Toh, 2004. The effect of local and general fatigue on knee proprioception. Arthroscopy, 20: 414-418. PMID: 15067282

19. Gribble, P.A. and J. Hertel, 2004. Effect of lowerextremity muscle fatigue on postural control. Arch. Phys. Med. Rehabil., 85: 589-592.

20. Granata, K.P. and G.P. Slota, 2004. Influence of fatigue in neuromuscular control of spinal stability. $\quad$ PMC, 46: 81-91. http://www.ncbi.nlm.nih.gov/pmc/articles/PMC16 33714/

21. Barrack, R.L., H.B. Skinner and S.L. Buckley, 1989. Proprioception in the anterior cruciate deficient knee. Am. J. Sports Med., 17: 1-6. http://ajs.sagepub.com/content/17/1/1.short

22. Renstrom, P.A.F.H. and P. Kannus, 2000. Prevention of Injuries in Endurance Athletes. In: Blackwell Science, Shephard, R.J. and P.O. Astrand (Eds.), 2nd Edn., Endurance in Sport Oxford.

23. Murphy, D.F., D.A.J. Connolly and B.D. Beynnon, 2003. Risk factors for lower extremity injury: A review of the literature. Br. J. Sports Med., 37: 13-29. PMCID: PMC1724594

24. Rozzi, S., P. Yuktanandana, D. Pincivero and S.M. Lephart, 2000. Role of Fatigue on Proprioception and Neuromuscular Control. In: Lephart, Proprioception and Neuromuscular Control in Joint Stability Champaign, S.M. and F.H. Fu (Eds.). Human Kinetics, IL., pp: 375-383.
25. Reilly, T. and G.A. Brooks, 1986. Exercise and the circadian variation in body temperature measures. Internl. J. Sports Med., 7: 358-362. http://www.ncbi.nlm.nih.gov/pubmed/3804546

26. Palastanga, N., D. Field and R. Soames, 2006. Anatomy and Human Movement: Structure and Function. 5th Edn., Butterworth Heinemann, London, ISBN: 0750688149, pp: 300-323.

27. Adlerton, A.K., U. Moritz and R. Moe-Nilssen, 2003. Force plate and accelerometer measures for evaluating the effect of muscle fatigue on postural control during one-legged stance. Physiother. Res. Int., 8: 187-199. DOI: 10.1002/pri.289

28. Woods, C., R. Hawkins, M. Hulse and A. Hodson, 2003. The football association medical research Programme: An audit of injuries in professional football: An analysis of ankle sprains. Br. J. Sports Med., 37: 233-238. PMCID: PMC1724634

29. Balduini, F., J.J. Vergso, J.S. Torg and E. Torg, 1987. Management and rehabilitation of ligamentous injuries to the ankle. Sports Med., 4: 364-380. PMID: 3313619

30. Hawkins, R.D., M.A. Hulse, C. Wilkinson, A. Hodson and M. Gibson, 2001. The association football medical research programme: An audit of injuries in professional football. Br. J. Sports Med., 35: 43-47. http://www.ncbi.nlm.nih.gov/pmc/articles/PMC172 4575/

31. Nyland, J.A., R. Shapiro, R.L. Stine, T.S. Horn and M.L. Ireland, 1994. Relationship of fatigued run and rapid stop to ground reaction forces, lower extremity kinematics and muscle activation. J. Orthopaed. Sports Phys. Ther., 20: 132-137.

32. Gleeson, N.P., T. Reilly, H. Mercer, S. Rakowski and D. Ress, 1998. Influence of acute endurance activity on leg neuromuscular and musculoskeletal performance. Med. Sci. Sports Exerc., 30: 596-608. http://cat.inist.fr/?aModele $=$ afficheN\&cpsidt $=2241$ 938

33. Zhou, S., M.J. McKenna, D.L. Lawson, W.E. Morrison and I. Fairweather, 1996. Effect of fatigue and sprint training on electromechanical delay of knee extensor muscles. Eur. J. Applied Physiol. Occupat. $\quad$ Physiol., 72: 410-416. http://works.bepress.com/shi_zhou/43/

34. Wojtys, E.W., B.B. Wylie and L.J. Huston, 1996. The effects of muscle fatigue on neuromuscular function and anterior tibial translation in healthy knees. Am. J. Sports Med., 24: 615-621. http://www.ncbi.nlm.nih.gov/pubmed/8883681 
35. Hewett, T.E., T.N. Lindenfeld, J.V. Riccobene and F.R. Noyes, 1999. The effect of neuromuscular training on the incidence of knee injury in female athletes: A prospective study. Am. J. Sports Med., 27: 699-705. PMID: 10569353

36. Matt, G. and W.J. Colin, 2007. The influence of soccer-specific fatigue on functional stability. Phys. Ther. Sport, 8: 185-190.

37. Girard, O., G. Lattier, J.P. Micallef and G.P. Millet, 2006. Changes in exercise characteristics, maximal voluntary contraction and explosive strength during prolonged tennis playing. Br. J. Sports Med., 40: 521-526. http://bjsm.bmj.com/cgi/content/abstract/40/6/521

38. Joseph B. Myers, Kevin M. Guskiewicz, Robert A. Schneider, and William E. Prentice, 1999. Proprioception and neuromuscular control of the shoulder after muscle fatigue. J. Athl. Train, 34: 362-367. PMCID: PMC1323348

39. Hornery, D.J., D. Farrow, I. Mujika, W. Young, 2007. Fatigue in tennis. Mechanisms of fatigue and effect on performance. Sports Med., 37: 199-212. http://www.ingentaconnect.com/content/adis/smd/2 007/00000037/00000003/art00002

40. Cerulli, G., D. Benoit, A. Caraffa and F. Ponteggia, 2001. Proprioceptive training and prevention of anterior cruciate ligament injuries in soccer. $\mathrm{J}$. Orthopaed. Sports Phys. Ther., 31: 655-660. http://www.ncbi.nlm.nih.gov/pubmed/11720298

41. Myklebust, G., L. Engebretsen, I. Braekken, A. Skjolberg, O. Olsen and R. Bahr, 2002. Prevention of anterior cruciate ligament injuries in female team handball players: A prospective intervention study over three seasons. Clin. J. Sport Med., 13: 71-78.

42. Vuillerme, N., N. Pinsault and J. Vaillant, 2005. Postural control during quiet standing following cervical muscular fatigue: Effects of changes in sensory inputs. Neurosci. Lett., 378: 135-139. DOI: 10.1016/j.neulet.2004.12.024

43. Schieppati, M., 2003. Neck muscle fatigue affects postural control in man. Neuroscience, 121: 277-285.

44. Adam, J., S.W.P. Berg, 2007. Fatigue-induced adaptive changes of anticipatory postural adjustments. Exp. Brain Res., 178: 49-61. http://www.ncbi.nlm.nih.gov/pubmed/17039358

45. Byars, A., M. Greenwood, L. Greenwood and W. Simpson, 2006. The Effectiveness of a PreExercise Performance Drink (PRX) on indices of maximal cardiorespiratory fitness. J. Int. Soc. Sports Nutr., 3: 56-59. http://www.ncbi.nlm.nih.gov/pmc/articles/PMC212 9156/
46. Carvajal-Sancho, A. and J. Moncada-Jiménez, 2005. The acute effect of an energy drink on physical and cognitive performance of male athletes. Kinesiologia Slovenica, 11: 5-16.

47. Goudarzi, A., 2002. Reliability and validity of RAST test in assessment of young male glycolytic power. M.Sc. Thesis, Department of Physical Education and Sport Sciences, Faculty of the Human Sciences, Hamadan University, Hamadan, Iran.

48. Greig, M. and C. Walker-Johnson, 2007. The influence of soccer-specific fatigue on functional stability. Phys. Ther. Sports, 8: 185-90. http://www.physicaltherapyinsport.com/article/S14 66-853X(07)00029-6/abstract

49. Pincivero, D.M., S.M. Lephart and T. Henry, 1995. Learning effects and reliability of the Biodex Stability System. Abst. J. Athl. Train, 30: S35

50. Testerman, C. and R.V. Griend, 1999. Evaluation of ankle instability using the biodex stability systems. Foot Ankle Int., 20: 317-321. http://www.ncbi.nlm.nih.gov/pubmed/10353771

51. Vachon, A.J., R.D. Bassett and S. Clarke, 1999. Validity of the heart rate deflection point as a predictor of lactate threshold during running. J. Applied Physiol., 87: 452-459. http://www.ncbi.nlm.nih.gov/pubmed/10409607

52. Zacharogiannis, E., G. Paradisis and S. Tziortzis, 2004. An evaluation of tests of anaerobic power and capacity. Med. Sci. Sports Exerc., 36: S116.

53. Graham, D., D. Lamb, G. Stephenson, J. Bangsbo and C. Juel, 2006. Point: Counterpoint: Lactic acid accumulation is an advantage/disadvantage during muscle activity. J. Applied Physiol., 100: 1410-1414.

54. Juel, C. and A.P. Halestrap, 1999. Lactate transport in skeletal muscle-role and regulation of the monocarboxylate transporter. J. Physiol., 517: 633-642. http://www.ncbi.nlm.nih.gov/pubmed/10358105

55. Myklebust, G., L. Engebretsen, I. Braekken, A. Skjolberg, O. Olsen and R. Bahr, 2002. Prevention of anterior cruciate ligament injuries in female team handball players: A prospective intervention study over three seasons. Clin. J. Sports Med., 13: 71-78. http://www.ncbi.nlm.nih.gov/pubmed/12629423 